\title{
Prediction of histologic differentiation grade of hepatocellular carcinoma by magnetic resonance imaging with hepatospecific contrast agent
}

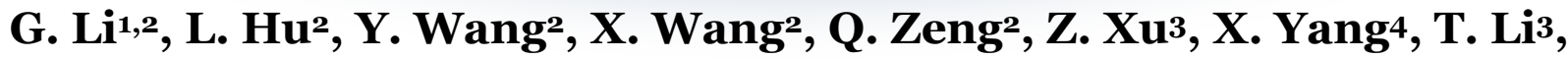 \\ H. Sun ${ }^{*}$, H. Shi ${ }^{*}$ \\ ${ }^{1}$ Department of Radiology, Shandong Qianfoshan Hospital, Cheeloo College of Medicine, Shandong University, Jinan, \\ Shandong, 250014, P.R. China \\ ${ }^{2}$ Department of Radiology, The First Affiliated Hospital of Shandong First Medical University, Jinan, Shandong, \\ 250014, P.R. China \\ ${ }^{3}$ Department of General Surgery, The First Affiliated Hospital of Shandong First Medical University, Jinan, Shandong, \\ 250014, P.R. China \\ ${ }^{4}$ Department of Pathology, The First Affiliated Hospital of Shandong First Medical University, Jinan, Shandong, \\ 250014, P.R. China
}

\section{- Original article}

*Corresponding authors:
Dr. Hongjun Sun,
E-mail:
$\quad$ qysunhongjun@163.com
Dr. Hao Shi,
E-mail:
$\quad$ hansenschie@163.com
Revised: July 2020
Accepted: August 2020
Int. J. Radiat. Res., October 2021;
19(4): 791-799
DOI: 10.29242/ijrr.19.4.791

*Both authors equally contributed to this paper and thus shared the co-corresponding authorship.

\begin{abstract}
Background: To evaluate the predictive value at the hepatobiliary phase (HBP) magnetic resonance imaging (MRI) after gadobenate dimeglumine (GdBOPTA) injection for histologic grades of hepatocellular carcinoma (HCC). Materials and Methods: HCC patients who underwent Gd-BOPTA-enhanced MRI were analyzed and divided into four histologic grades of differentiation based on Edmonson-Steiner grade. Besides, HCC tumor differentiations grade I, II and III, IVwere defined as well-differentiated (WD), moderatelydifferentiated (MD), poorly-differentiated (PD), respectively. The clinical, pathological and imaging features of patients were assessed. Receiver operating characteristic $(R O C)$ curve was to evaluate the significance between WD/MD HCC and PD HCC using the apparent diffusion coefficient (ADC), relative intensity ratio (RIR) of T1WI and T2WI (T1RIR, T2RIR), contrastenhancement ratio in arterial phase (CERA) and contrast-noise-ratio (CNR) in

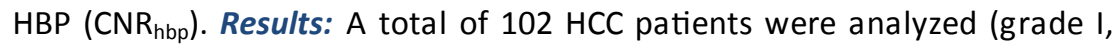
$n=19$; grade II, $n=30$; grade III, $n=30$; grade IV, $n=23$ ). There was no statistical difference regarding to the pathological and clinical features among

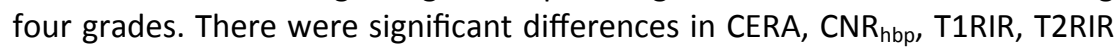
and ADC values among the four HCC grades (all $P<0.05$ ). Based on ROC curve, comparison of the five quantitative MRI parameters indicated ADC (sensitivity $84.8 \%$, specificity $82.6 \%$, AUC 0.862 ) and CNR $_{\text {hbp }}$ value (sensitivity $78.5 \%$, specificity 91.3\%, AUC 0.916) could significantly distinguish the HCC histological grade. Conclusions: The ADC and/or $\mathrm{CNR}_{\mathrm{hbp}}$ value in HBP-MRI after GD-BOPTA injection might be as the significant quantitative parameters to distinguish $\mathrm{HCC}$ histological grade.
\end{abstract}

Keywords: Hepatocellular carcinoma, gadobenate dimeglumine, magnetic resonance imaging, apparent diffusion coefficient, contrast noise ratio.

\section{INTRODUCTION}

Hepatocellular carcinoma (HCC) is a common primary malignant liver tumor, which often occurs in patients with chronic hepatic parenchymal disease. Its incidence rate and mortality rate of are high in worldwide, especially in Africa and Asia (1). HCC is the second highest mortality rate for male tumor-related diseases. There are approximately 
750,000 new HCC patients worldwide each year, and the prevalence rate for males is 2-4 times higher than for females $(2,3)$. Surgical resection or liver transplantation are the principal treatment for HCC (4), which have made continuous progress. However, for cure and long-term survival of HCC, the high recurrence rate is still the biggest shortcoming of surgery.

It is very important to evaluate the prognosis of HCC patients. After liver transplantation and resection, the histologic grade is one the primary predictive factor of recurrence and survival in HCC patients (5). Before operation, the histological grade of is the main parameter of treatment planning for HCC. As we known, $1 \mathrm{~cm}$ around the lesion is the tumor-free margin in surgical resection that is internationally recognized (6). Furthermore, if HCC patients with high pathological grade before surgery, the resection range of lesion could be expanded ensuring not affect the liver function after resection. Previously, Shi et al. have reported the recurrence rate of HCC was markedly decreased when extending the tumor-free margin to $2 \mathrm{~cm}$ (7). The preoperative imaging plays a vital role for the assessment of well-differentiated (WD), moderately-differentiated (MD) and poorly-differentiated (PD) HCC (8). Nevertheless, it is often challenging to determine which type of tumor differentiation it is, due to different histological grades of HCCs usually have overlapping imaging appearances for any given magnetic resonance imaging (MRI) features.

Gadobenate dimeglumine (Gd-BOPTA), as one of gadolinium-based paramagnetic contrast agents, could take the acquisition of images in the delayed T1-weighted liver-specific phase after injection in hepatobiliary phase (HBP) about 1.5-2 h $(9,10)$. There were several reports have indicated Gd-BOPTA is superior to the conventional extracellular MRI contrast regarding to predict HCCs histological grade that defined by World Health Organization (11-13). However, few studies investigating the correlation between the enhancement patterns on Gd-EOB-DTPA enhanced MRI scans and the HCCs histological grade based on Edmondson-Steiner system have been conducted. Furthermore, previous reports had attempted to combine MRI findings with HCCs histological grade using gadolinium based liver-specific contrast agents or apparent diffusion coefficient (ADC) enhanced MRI, but the consensus was not obtained $(14,15)$. Most those studies are based on the signal intensity (SI) of the surrounding liver parenchyma, but ignore the function of the liver parenchyma around the lesion. Therefore, erector spinae muscle may be more suitable in clinic. Thus, the aim of the present study was to investigate the value of Gd-BOPTA-enhanced dynamic MRI in distinguishing between the 4 different HCCs histological grade based on Edmondson-Steiner system in patients using the SI of the erector spinae as internal reference.

\section{MATERIALS AND METHODS}

\section{Patients}

The HCC patients who were performed with liver MRI using Gd-BOPTA in a single center from January 2016 to January 2020 were retrospectively analyzed. This study was approved by the institutional review board of our hospital (No. 2020-S303). Informed consent of patient was waived because of the retrospective study nature. All patients underwent surgical resection after MRI examinations. The inclusion criteria were: (1) patients underwent Gd-BOPTA-enhanced MRI examinations before surgery within 4 weeks; (2) tumor size of $10 \mathrm{~mm}$ or greater. The exclusion criteria were: (1) Child-Pugh class $\mathrm{C}$ cirrhosis, liver function was severely damaged; (3) poor image quality (e.g. artifact leading to poor measurement of lesions).

\section{MRI technique}

Images were acquired in the routine clinical workup on a 3.0 T GE Healthcare Discovery 750 (GE Signa, Milwaukee, WI, USA) with an 8-channel body phased-array coil. The patients were required to fast and abstain from food and water $4 \mathrm{~h}$ before MRI. The magnetic resonance (MR) images were acquired using the following sequences: (1) Liver acquisition with volume acceleration (LAVA) Flex, T1-weighted

Int. J. Radiat. Res., Vol. 19 No. 4, October 2021 
gradient-echo in-phase sequence (T1WI) $(\mathrm{TR}=$ $3.7 \mathrm{~ms}, \mathrm{TE}=1.2-2.3 \mathrm{~ms}$, hold breath $=12-15 \mathrm{~s}$, slice thickness $=5 \mathrm{~mm}$, matrix size $=260 \times 224$, field of view $($ FOV $)=340 \times 340 \mathrm{~mm}$ ); (2) periodically rotated overlapping parallel lines with enhanced reconstruction (PROPELLER), a respiration-triggered T2-weighted fat-suppression turbo spin-echo sequence $(\mathrm{TR}=4000 \mathrm{~ms}, \mathrm{TE}=72 \mathrm{~ms}$, matrix size $=320 \times 320$, slice thickness $=6 \mathrm{~mm}$, slice gap $=$ $0.5 \mathrm{~mm}, \mathrm{FOV}=320 \times 320 \mathrm{~mm}$ ) and a respirationtriggered T2-weighted turbo spin-echo sequence $(\mathrm{TR}=4000 \mathrm{~ms}, \mathrm{TE}=72 \mathrm{~ms}$, matrix size $=$ $320 \times 320$, slice thickness $=6 \mathrm{~mm}$, slice gap $=0.5$ $\mathrm{mm}$, FOV $=320 \times 320 \mathrm{~mm}$ ); (3) a diffusionweighted imaging (DWI) with the respiratory triggered sensitivity encoding technique $(\mathrm{TR}=$ $8500 \mathrm{~ms}, \mathrm{TE}=57 \mathrm{~ms}$, matrix size $=256 \times 192$, slice thickness $=6 \mathrm{~mm}$, slice gap $=0.5 \mathrm{~mm}$, FOV $=380 \times 380 \mathrm{~mm}, \mathrm{~b}=800 \mathrm{~s} / \mathrm{mm}^{2}$ ). For enhanced dynamic study, Gd-BOPTA was injected at a dose of $0.1 \mathrm{~mL} / \mathrm{kg}(0.2 \mu \mathrm{mol} / \mathrm{kg})$, following $20 \mathrm{~mL}$ saline was immediately injected at a rate of $2 \mathrm{~mL} / \mathrm{s}$. Dynamic imaging, including the arterial (25 s), portal venous (60 s), and equilibrium phases $(180 \mathrm{~s})$, and the HBP (90-120 min), was performed using LAVA Flex sequence.

\section{Imaging interpretation}

All MRI images were reviewed by 2 experienced radiologists blinded to the histopathological results who had more than 10 years of experience in liver imaging. Quantitative analysis of each HCC lesion was performed to derive potential objective pathophysiological information from the following six parameters: apparent diffusion coefficient (ADC), relative intensity ratio (RIR) of T2WI and T1WI (T1RIR, T2RIR), the contrast-noise-ratio in $\mathrm{HBP}$ ( $\mathrm{CNR}_{\mathrm{hbp}}$ ), contrast-enhancement ratio in arterial phase (CERA). The ADC value in the background liver parenchyma was measured by placing manually defined ROI using the T2-weighted MRI sequence. The standard deviation of SI of the surrounding noise $\left(\mathrm{SD}_{\text {noi }}\right)$, liver parenchyma around the lesions ( $\mathrm{SI}_{\mathrm{hep}}$ ), erector spinae ( $\mathrm{SI}_{\mathrm{mus}}$ ) and signal intensities of lesions ( $\mathrm{SI}_{\text {les}}$ ) of each
HCC lesion were measured with regions of interest (ROIs) ROIs on precontrast, arterial phases (AP) and HBP images on the corresponding unenhanced T1WI. The ROI was measured 3 times at the same section and approximately $0.5-1.0 \mathrm{~cm}^{2}$ maximum oval or round, deliberately avoiding of necrosis cystic degeneration, hemorrhagic and blood vessels area. The RIR, CNR $\mathrm{hbp}_{\text {and }}$ CERA were defined as following: $\mathrm{RIR}=\mathrm{SI}_{\text {les }} / \mathrm{SI}_{\text {hep }} ; \mathrm{CNR}_{\mathrm{hbp}}=$ $\left(\mathrm{SI}_{\text {les }}-\mathrm{SI}_{\text {mus }}\right) / \mathrm{SD}_{\text {noi }} ; \mathrm{CERA}=\left[\left(\mathrm{SI}_{\text {arterial }}-\mathrm{SI}_{\text {precontrast }}\right) /\right.$ $\left.S I_{\text {precontrast }}\right] \times 100 \%(16)$, respectively.

\section{Pathological analysis and histological grade}

All HCC specimens were obtained through surgical resection. Representative areas of each tumor and the liver parenchyma were then selected for a microscopic analysis. The tissue was fixed with $10 \%$ formaldehyde, then embedded in paraffin, sliced into $4 \mu \mathrm{m}$ sections for hematoxylin-eosin staining. All sample tissues were evaluated by a pathology resident and then confirmed by an attending pathologist, who were blinded to the MRI findings. The pathologic reports included the histologic grade, tumor size, capsule, lipid, hemorrhage, necrosis, tumor thrombi, cirrhosis and location of the HCC. On the basis of the Edmonson-Steiner grade, tumor differentiation was divided into I, II, III, IV grade (17). Specifically, Edmondson-Steiner grade I was defined as WD, grade II and III as MD, and grade IV as PD (14).

\section{Statistical analysis}

The SPSS software 20.0 (IBMSPSS, Chicago, IIl) were used to analyze the data. Categorical variables were compared by chi-square or Fisher's exact test. Continuous variables were presented as mean value \pm standard deviation (minimal Value-maximal value) and compared by Mann-Whitney U test. The Mann-Whitney U test was also used for group comparisons regarding to quantitative MRI measurements according to the HCC histological grades. In addition, receiver operating characteristic (ROC) curve were used to analyze the significance in distinguishing WD/MD HCC from PD HCC using

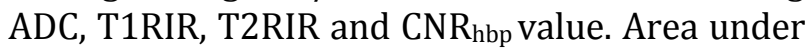
the curve (AUC) based on optimum thresholds 
for variable parameters were calculated. The cutoff values of each quantitative parameter were chosen that provided optimal sensitivity and specificity. $\quad \mathrm{P}<0.05$ was considered statistically significant.

\section{RESULTS}

\section{Clinical and pathological features of the HCC patients}

The baseline clinical characteristics of 102 HCC patients were shown in table 1, including 81 men and 21 women, which translates to a male-to-female ratio 3.86:1. The median age was $56.36 \pm 9.58$ years (range, 28-78 years). The diameter of all lesions ranged from 1 to $18 \mathrm{~cm}$, with a mean (SD) of $4.78(3.18) \mathrm{cm}$. According to Edmonson-Steiner grade, all HCC patients were divided into grade I ( $n=19)$, II $(n=30)$, III $(n=30)$ and IV $(n=23)$ groups. There was no statistical difference in terms of age, sex, lesion location, tumor size, Child-pugh class, and $\alpha$-fetoprotein (AFP) level among different differentiated HCC groups, which might indicate there was no significant correlation between tumor grade and those clinical characteristic (all $\mathrm{P}>0.05$ ). In particularly, tumor grade had no correlation to tumor size, that similar to one previous report (14).

The necrosis rate of grade I-IV groups were $42.1 \%, 53.3 \%, 66.7 \%$ and $82.6 \%$, respectively, which increased with the increase of grade with statistically significant difference $\left(\chi^{2}=8.55\right.$, $\mathrm{P}=0.036)$. There were significant differences in terms of tumor capsule rate $\left(\chi^{2}=10.93, \mathrm{P}=0.01\right.$; especially grade II vs. IV, $\mathrm{P}=0.007)$ and emboli rate of $\mathrm{HCC}\left(\chi^{2}=14.28, \mathrm{P}=0.001\right.$; specially grade I vs. IV, $\mathrm{P}=0.001)$ among grade I-IV groups. While, when compared the pathological of the background liver parenchyma, the results indicated there was no significant differences regarding the presence of hemorrhage, fatty change, and liver cirrhosis among different differentiated HCC groups (table 2, all $\mathrm{P}>0.05$ ). This can be thought of as the malignant degree of HCC increased, and it was more aggressive, so the damage to the adjacent into blood vessels improved.

\section{Relationship between quantitative MRI parameters and histological grade}

As detailed in table 3, we found CERA, $\mathrm{CNR}_{\mathrm{hbp}}$, T1RIR, T2RIR and ADC values showed significant differences among HCC grade I-IV groups (all $\mathrm{P}<0.05$ ). Furthermore, the pairwise comparison of HCC grade I-IV groups in term of each quantitative MRI measured parameters were analyzed (table 4). The results showed there was no significant difference in term of CERA, $\mathrm{CNR}_{\mathrm{hbp}}$, T1RIR and T2RIR between grade II and III (all $\mathrm{P}>0.05$ ), while there were significant differences in terms of five parameters between grade I and IV (all $\mathrm{P}<0.05$ ). These differences between the grades corresponded to Edmondson-Steiner grading system: grade I was defined as WD, grade II and III as MD, and grade IV as PD (14).

\section{ROC analysis}

Considering previous study has found the quantitative parameters were less reliable to differentiating PD HCC from WD/MD HCC (18). Thus, the cut-off values, sensitivity, specificity, and AUC of the five parameters (CERA, CNRhbp, T1RIR, T2RIR and ADC) were then calculated (table 5). Comparison of the five quantitative MRI parameters indicated $\mathrm{CNR}_{\mathrm{hbp}}$ and $\mathrm{ADC}$ value might be the two important parameters for distinguish histological grade. The ROC curve for these $\mathrm{ADC}$ value and $\mathrm{CNR}_{\mathrm{hbp}}$ was shown in figure 1. The cut-off value for ADC in differentiating between PD HCC and WD/MD HCC was $0.771 \times$ $10^{-3} \mathrm{~mm}^{2} / \mathrm{s}$ (sensitivity $84.8 \%$, specificity $82.6 \%$, AUC 0.862), and for CNR $\mathrm{CNb}_{\mathrm{hb}}$ was 40.5 (sensitivity $78.5 \%$, specificity $91.3 \%$, AUC 0.916). In addition, the two typical cases were shown in figure 2-3. 
Table 1. The baseline clinical characteristics of the $102 \mathrm{HCC}$ patients.

\begin{tabular}{|c|c|c|c|c|c|c|c|}
\hline Characteristic & Total $(n=102)$ & Grade I (n=19) & Grade II $(n=30)$ & Grade III $(n=30)$ & Grade IV $(n=23)$ & $F / \chi^{2}$ & $\mathbf{P}$ \\
\hline Age (year) & $56.36 \pm 9.58$ & $61.68 \pm 10.79$ & $54.23 \pm 8.29$ & $54.93 \pm 7.93$ & $56.61 \pm 10.92$ & 2.819 & 0.043 \\
\hline Sex (n) & & & & & & 1.947 & 0.584 \\
\hline Male & 81 & 16 & 23 & 22 & 20 & & \\
\hline Female & 21 & 3 & 7 & 8 & 3 & & \\
\hline Lesion Location (n) & & & & & & 0.584 & 0.900 \\
\hline Left lobe & 27 & 6 & 8 & 8 & 5 & & \\
\hline Right lobe & 71 & 12 & 20 & 22 & 17 & & \\
\hline Both lobes & 4 & 1 & 2 & 0 & 1 & & \\
\hline Tumor size $(\mathrm{cm})$ & $4.78 \pm 3.18$ & $4.84 \pm 3.74$ & $4.28 \pm 2.65$ & $4.50 \pm 3.60$ & $5.48 \pm 2.64$ & 0.700 & 0.555 \\
\hline Child-pugh & & & & & & 2.480 & 0.479 \\
\hline A & 87 & 18 & 25 & 24 & 20 & & \\
\hline B & 15 & 1 & 5 & 6 & 3 & & \\
\hline Pathogenic features & & & & & & 4.653 & 0.199 \\
\hline HBV $(n)$ & 95 & 18 & 28 & 29 & 20 & & \\
\hline HCV (n) & 7 & 1 & 2 & 1 & 3 & & \\
\hline $\operatorname{AFP}(\mathrm{ng} / \mathrm{mL})(\mathrm{n})$ & & & & & & 16.371 & 0.060 \\
\hline$<10$ & 40 & 10 & 16 & 8 & 6 & & \\
\hline $10-100$ & 23 & 4 & 7 & 9 & 3 & & \\
\hline $101-400$ & 9 & 4 & 1 & 5 & 4 & & \\
\hline$>400$ & 25 & 1 & 6 & 8 & 10 & & \\
\hline
\end{tabular}

$\mathrm{HCC}$, hepatocellular carcinoma; HBV, hepatitis B virus; HCV, hepatitis C virus; AFP, $\alpha$-fetoprotein.

Table 2. Pathological features of HCC patients.

\begin{tabular}{|c|c|c|c|c|c|c|}
\hline Characteristics (n, \%) & Grade I (n=19) & Grade II (n=30) & Grade III (n=30) & Grade IV (n=23) & $\mathbf{\chi}^{\mathbf{2}}$ & $\mathbf{P}$ \\
\hline Necrosis & $8(42 \%)$ & $16(53.3 \%)$ & $20(66.7 \%)$ & $19(83 \%)$ & 8.55 & 0.036 \\
\hline Bleeding & $8(42 \%)$ & $7(23.3 \%)$ & $8(26.7 \%)$ & $12(52 \%)$ & 6.15 & 0.105 \\
\hline Tumor capsule & $17(89 \%)$ & $29(96.7 \%)$ & $22(73.3 \%)$ & $15(65 \%)$ & 10.93 & 0.01 \\
\hline Fatty change & $12(63 \%)$ & $9(30 \%)$ & $12(40 \%)$ & $8(35 \%)$ & 5.75 & 0.125 \\
\hline Tumor emboli & 0 & $3(10 \%)$ & $5(16.7 \%)$ & $10(43.5 \%)$ & 14.28 & 0.001 \\
\hline Cirrhosis & $16(84 \%)$ & $21(70 \%)$ & $23(76.7 \%)$ & $18(78 \%)$ & 1.32 & 0.73 \\
\hline
\end{tabular}

Table 3. Comparisons of different imaging parameters among 4 histological grade according to Edmondson-Steiner Grade (HCCs, $\mathrm{n}=102$ ).

\begin{tabular}{|c|c|c|c|c|c|c|}
\hline Measurements & Grade I( $\mathbf{n}=\mathbf{1 9})$ & Grade II $\mathbf{( n = 3 0 )}$ & Grade III $\mathbf{( n = 3 0 )}$ & Grade IV $(\mathbf{n}=\mathbf{2 3})$ & $\mathbf{F}$ & $\mathbf{P}$ \\
\hline CERA & $1.32 \pm 0.54$ & $1.23 \pm 1.25$ & $0.92 \pm 0.63$ & $0.63 \pm 0.26$ & 3.480 & 0.019 \\
\hline CNR $_{\mathbf{h b p}}$ & $96.63 \pm 14.89$ & $51.37 \pm 17.31$ & $47.43 \pm 12.57$ & $25.13 \pm 13.22$ & 85.169 & $<0.001$ \\
\hline T1RIR & $0.86 \pm 0.05$ & $0.81 \pm 0.12$ & $0.74 \pm 0.12$ & $0.70 \pm 0.80$ & 11.12 & $<0.001$ \\
\hline T2RIR & $1.21 \pm 0.30$ & $1.79 \pm 0.56$ & $1.79 \pm 0.38$ & $1.70 \pm 0.39$ & 8.914 & $<0.001$ \\
\hline ADC $\left(\mathbf{m m}^{\mathbf{2}} / \mathbf{s} \times \mathbf{1 0}^{-\mathbf{3}}\right)$ & $1.16 \pm 0.21$ & $0.94 \pm 0.16$ & $0.84 \pm 0.21$ & $0.69 \pm 0.14$ & 24.43 & $<0.001$ \\
\hline
\end{tabular}

T1RIR, relative intensity ratio (RIR) of T1WI; T2RIR, relative intensity ratio (RIR) of T2WI; CERA, contrast-enhancement ratio in aterial phase; CNRhbp, contrast noise ratio in HBP; ADC, apparent diffusion coefficient. 
Table 4. Pair comparison results of CERA, CNRhbp, T1RIR, T2RIR and ADC of each pathologically differentiated group.

\begin{tabular}{|c|c|c|c|c|}
\hline Parameters & Grade & I & II & III \\
\hline CERA & I & 1.00 & 0.128 & $<0.001$ \\
\hline & II & & 0.779 & 0.088 \\
\hline & III & & & 0.160 \\
\hline CNR $_{\text {hbp }}$ & I & $<0.001$ & $<0.001$ & $<0.001$ \\
\hline & II & & 0.144 & 0.002 \\
\hline & III & & & 0.624 \\
\hline T1RIR & I & 0.223 & $<0.001$ & $<0.001$ \\
\hline & II & & 0.144 & 0.002 \\
\hline & III & & & 0.624 \\
\hline T2RIR & I & $<0.001$ & $<0.001$ & $<0.001$ \\
\hline & II & & 1.000 & 0.976 \\
\hline & III & & & 0.935 \\
\hline ADC & I & $<0.001$ & $<0.001$ & $<0.001$ \\
\hline & II & & 0.04 & $<0.001$ \\
\hline & III & & & 0.004 \\
\hline
\end{tabular}

T1RIR, relative intensity ratio (RIR) of T1WI; T2RIR relative intensity ratio (RIR) of T2WI; CERA, contrastenhancement ratio in aterial phase; CNRhbp, contrast noise ratio in HBP; ADC, apparent diffusion coefficient.
Table 5. Receiver operating characteristic (ROC) curve analysis.

\begin{tabular}{|c|c|c|c|c|c|}
\hline Parameters & $\begin{array}{c}\text { AUC } \\
(95 \% \mathbf{C l})\end{array}$ & $\begin{array}{c}\text { Youden } \\
\text { index }\end{array}$ & $\begin{array}{c}\text { Cut-off } \\
\text { value }\end{array}$ & Sensitivity & Specificity \\
\hline ADC & $\begin{array}{c}0.862 \\
(0.781-0.944)\end{array}$ & 0.674 & $\begin{array}{c}0.771 \times 10^{-3} \\
\mathrm{~mm}^{2} / \mathrm{s}\end{array}$ & $84.8 \%$ & $82.6 \%$ \\
\hline CNR $_{\text {hbp }}$ & $\begin{array}{c}0.916 \\
(0.851-0.980)\end{array}$ & 0.698 & 40.5 & $78.5 \%$ & $91.3 \%$ \\
\hline CER & $\begin{array}{c}0.697 \\
(0.600-0.794)\end{array}$ & 0.50 & 1.01 & $54 \%$ & $96 \%$ \\
\hline T1RIR & $\begin{array}{c}0.746 \\
(0.650-0.851)\end{array}$ & 0.48 & 0.73 & $70 \%$ & $78 \%$ \\
\hline T2RIR & $\begin{array}{c}0.451 \\
(0.334-0.568)\end{array}$ & 0.11 & 2.06 & $24 \%$ & $13 \%$ \\
\hline
\end{tabular}

T1RIR, relative intensity ratio (RIR) of T1WI; T2RIR, relative intensity ratio (RIR) of T2WI; CERA, contrast-enhancement ratio in aterial phase; CNRhbp, contrast noise ratio in HBP; ADC, apparent diffusion coefficient; AUC, Area under the curve, $\mathrm{Cl}$, confidence interval.

Figure 1. ROC curve plotting sensitivity (y-axis) and 1specificity ( $x$-axis) of quantitative yellow solid curve as the diagonal line represented the AUC of 0.50 . The blue and grey solid curve represented CNRhbp and ADC, respectively. parameters. The
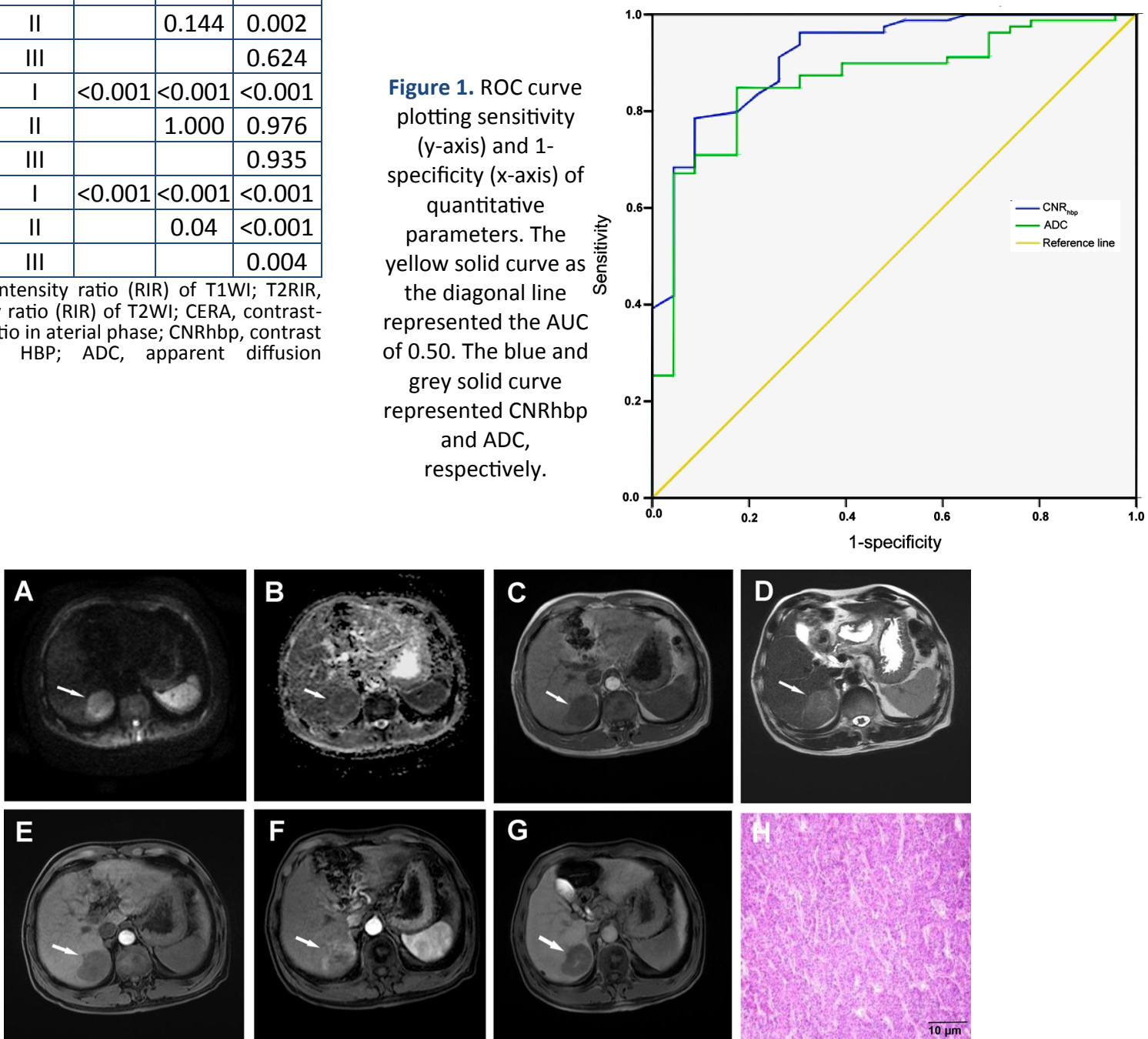

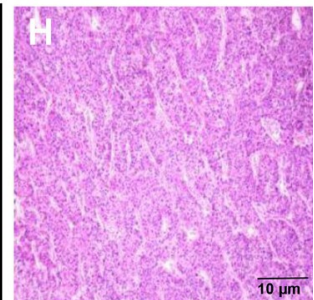

Figure 2. 56-year-old man with $5.0 \times 4.5 \mathrm{~cm}$ MD HCC in liver segment VI-VII. (A) High signal in DWI; (B) ADC map showed low signal, and the measured ADC value was $1.08 \times 10-3 \mathrm{~mm} 2 / \mathrm{s}$; (C) The lesions showed low signal in T1WI, and T1RIR was measured 0.69; (D) The lesions showed high signal in T2WI, and T2RIR was measured 1.59; (E) The lesion in T1WI fat-suppression showed low signal; (F) The lesion was significantly enhanced in arterial phase, and the CERA was measured as 2.75; (G) The hepatobiliary lesions showed low signal, and CNRhbp was 29; (H) HE staining showed grade II on the basis of the Edmonson-Steiner grade $(\times 100)$. 

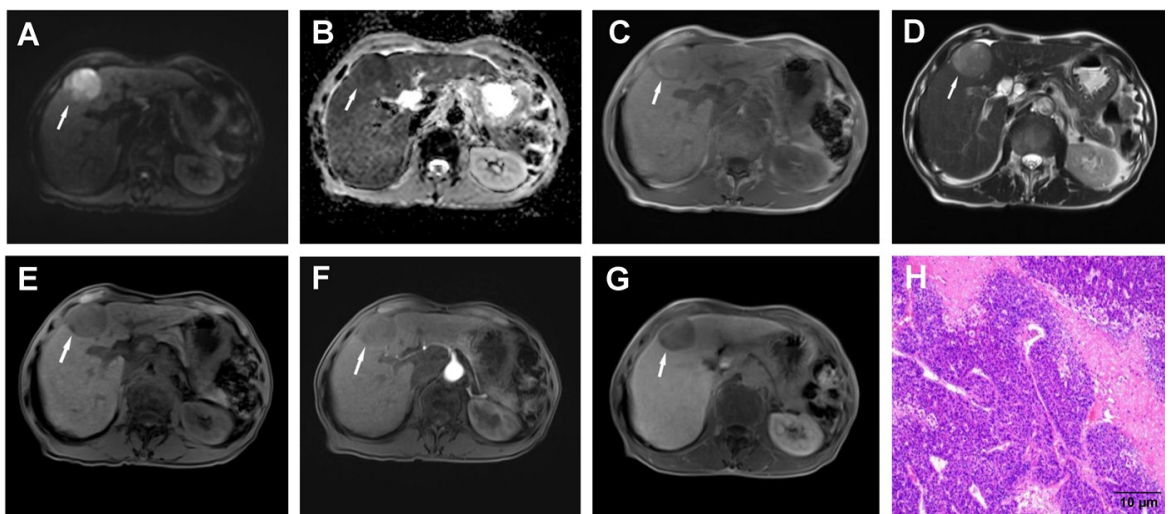

Figure 3. 71-year-old man with $5.3 \times 4.5 \mathrm{~cm}$ PD HCC in liver segment IV. (A) High signal in DWI; (B) ADC map showed low signal, and the measured ADC value was $0.632 \times 10-3 \mathrm{~mm} 2 / \mathrm{s}$; (C) The lesions showed mainly low signal in T1WI, and T1RIR was measured 0.63;

(D) The lesions showed high signal in T2WI, and T2RIR was measured 1.96; (E) The lesion in T1WI fat-suppression showed low signal; (F) The lesion was slightly enhanced in arterial phase, and the CERA was measured as 0.37; (G) The hepatobiliary lesions showed low signal, and CNRhbp was 11; (H) HE staining showed grade IV on the basis of the Edmonson-Steiner grade $(\times 100)$.

\section{DISCUSSION}

Gd-BOPTA-enhanced MRI could avoid long-term imaging follow-up or biopsy of small arterial enhancing-only nodules in liver cirrhosis patients, as it can reveal reliable predictors of HCC. Recently, several studies have shown that focal liver lesions could be obviously detect and diagnosed by Gd-BOPTA examination in $\operatorname{HBP}(10,14,19)$. In this study, the ROC curve analysis revealed $\mathrm{CNR}_{\mathrm{hbp}}$ value were the most significant quantitative MRI parameter to distinguish histological grade, which was similar to previous studies $(14,19)$. For example, Huang et al. (14). have reported the low CNR value in the HBP on Gd-BOPTA-enhanced MRI was related to the poorer histological differentiation of tumors, suggesting that preoperative quantitative MRI parameters could predict HCC histological grade. We speculated that this phenomenon may root in the fact that CNR value was affected more slightly by SI of erector spinae compared to the peripheral liver parenchyma.

Furthermore, several reports have indicated the ADC value from DWI could improve the function of MRI in HCC classification $(20,21)$. Our results were in accordance with previous researches indicating the ADC value of high-grade HCC was lower than that of low-grade HCC. The reason is that with the deterioration of HCC differentiation, tumor cells would be characterized by the increase of cellular density, nucleocytoplasmic ratio and Int. J. Radiat. Res., Vol. 19 No. 4, October 2021 intracellular organelles, and the thickening of cellular plates $(22,23)$. The transformations might lead to the shrinkage of intra- and extracellular space, following by the movement limitation of water molecules, finally result in diffusion restricted. The diffusivity of tissue was more limited, the ADC value would be lower (24).

Despite only partial statistical differences existed in comparisons between groups in terms of T1RIR and T2RIR, this study concluded that high-grade HCC showed lower RIRs on T1WI and higher RIRs on T2WI compared with low-grade HCCs, which were similar to previous studies $(19,25)$. According to previous reports $(26$, 27), this rule is generally followed in the multi-step evolution from degenerative nodules of cirrhosis to early hepatocellular carcinoma. However, the histopathological mechanism of the T1WI and T2WI signal changes observed among different pathological grades of HCC remains unclear. It is speculated that the more poor differentiation tumor cells are, the more water content or liquefactive necrosis are in the tissues.

Although, there only had significant difference regarding to CERA between grade I and IV, the enhancement rate of arterial phase has a downward trend with the increase of grading, which was similar to previous reports $(26,28)$. Yasuda et al. (29) suggested that the under hypoxia environment hypoxia inducible factor-1 (HIF-1) play an important role in the metastatic of liver cancer. For some PD HCCs, it may be 
supposed that tumor cells acquire metabolic profile of increased glycolysis via regulation of HIF-1 pathway, so that they enable to proliferate faster under hypoxia. That is to say, aerobic metabolizing was the main energy source for hypervascular HCC, while glycolysis was the main energy source for some PD HCCs with less arterial blood flow. Although our results suggested the quantitative MRI parameters might contribute to distinguish HCC grade, it was less reliable for differentiating WD/MD HCC from PD HCC. Further investigation is needed to clarify the real underlying molecular biology of these correlations.

This study had several limitations. Firstly, due to the nature of retrospective study, the sample size was relatively small. Secondly, there might have been a selection bias in our patient population. Thirdly, the patient population was enriched for HBV and Childs A liver dysfunction, which limits applicability of the results to a patients with more severe liver disease. Fourthly, the verification of the presence of contrast in the bile duct is also mandatory, because HBP can be delayed in cirrhotic patients. In future, a multicenter trial with larger number of patients would be desirable to confirm these findings.

\section{CONCLUSION}

In conclusion, the $\mathrm{ADC}$ and/or $\mathrm{CNR}_{\text {hbp }}$ value in HBP-MRI after GD-BOPTA injection might be as the significant quantitative parameters to distinguish HCC histological grade.

\section{Conflicts of interest: Declared none.}

\section{REFERENCES}

1. Dai Q, Zhang C, Yuan Z, Sun Q, Jiang Y (2020) Current discovery strategies for hepatocellular carcinoma therapeutics. Expert opinion on drug discovery, 15(2): 243 $-258$.

2. Torre LA, Bray F, Siegel RL, Ferlay J, Lortet-Tieulent J, Jemal A (2015) Global cancer statistics, 2012. CA: A Cancer Journal for Clinicians, 65(2): 87-108.
3. Cai Z and Liu Q (2019) Understanding the global cancer statistics 2018: Implications for cancer control. Science China Life sciences.

4. Schlachterman A, Craft WW, Jr., Hilgenfeldt E, Mitra A, Cabrera R (2015) Current and future treatments for hepatocellular carcinoma. World journal of gastroenterology, 21(28): 8478-8491.

5. Wang $F$, Numata $K$, Nakano $M$, Tanabe $M$, Chuma $M$, Nihonmatsu H, Nozaki A, Ogushi K, Luo W, Ruan L et al. (2020) Diagnostic value of imaging methods in the histological four grading of hepatocellular carcinoma. Diagnostics, 10(5).

6. Yang P, Si A, Yang J, Cheng Z, Wang K, Li J, Xia Y, Zhang B, Pawlik TM, Lau WY et al. (2019) A wide-margin liver resection improves long-term outcomes for patients with HBV-related hepatocellular carcinoma with microvascular invasion. Surgery, 165(4): 721-730.

7. Shi M, Guo RP, Lin XJ, Zhang YQ, Chen MS, Zhang CQ, Lau WY, Li JQ (2007) Partial hepatectomy with wide versus narrow resection margin for solitary hepatocellular carcinoma: A Prospective Randomized Trial. Annals of Surgery, 245(1): 36-43.

8. Huang $K$, Dong $Z$, Cai $H$, Huang $M$, Peng $Z$, Xu L, Jia $Y$, Song C, Li ZP, Feng ST (2019) Imaging biomarkers for well and moderate hepatocellular carcinoma: preoperative magnetic resonance image and histopathological correlation. BMC Cancer, 19(1): 364.

9. Cortis K, Liotta R, Miraglia R, Caruso S, Tuzzolino F, Luca A (2016) Incorporating the hepatobiliary phase of gadobenate dimeglumine-enhanced MRI in the diagnosis of hepatocellular carcinoma: increasing the sensitivity without compromising specificity. Acta Radiol, 57(8): 923931.

10. Reizine E, Amaddeo G, Pigneur F, Baranes L, Legou F, Mule S, Zegai B, Roche V, Laurent A, Rahmouni A, et al. (2018) Quantitative correlation between uptake of Gd-BOPTA on hepatobiliary phase and tumor molecular features in patients with benign hepatocellular lesions. European radiology, 28(10): 4243-4253.

11. Park Y, Kim SH, Kim SH, Jeon YH, Lee J, Kim MJ, Choi D, Lee WJ, Kim H, Koo JH, et al. (2010) Gadoxetic acid (Gd-EOBDTPA)-enhanced MRI versus gadobenate dimeglumine (Gd -BOPTA)-enhanced MRI for preoperatively detecting hepatocellular carcinoma: an initial experience. Korean Journal of Radiology, 11(4): 433-440.

12. Lebert $P$, Adens-Fauquembergue $M$, Azahaf $M$, Gnemmi $V$, Behal H, Luciani A, Ernst O (2019) MRI for characterization of benign hepatocellular tumors on hepatobiliary phase: the added value of in-phase imaging and lesion-to-liver visual signal intensity ratio. European Radiology, 29(11): 5742-5751.

13. Allen BC, Ho LM, Jaffe TA, Miller CM, Mazurowski MA, Bashir MR (2018) Comparison of visualization rates of LIRADS version 2014 major features with IV gadobenate dimeglumine or gadoxetate disodium in patients at risk for hepatocellular carcinoma. AJR American Journal of Roentgenology, 210(6): 1266-1272.

Int. J. Radiat. Res., Vol. 19 No. 4, October 2021 
14. Huang $X$, Xiao $Z$, Zhang $Y$, Lin $N$, Xiong $M$, Huang $X$, Chen $Q$, Cao D (2018) Hepatocellular carcinoma: Retrospective evaluation of the correlation between gadobenate dimeglumine-enhanced magnetic resonance imaging and pathologic grade. Journal of Computer Assisted Tomography, 42(3): 365-372.

15. Kakite S, Dyvorne H, Besa C, Cooper N, Facciuto M, Donnerhack C, Taouli B (2015) Hepatocellular carcinoma: short-term reproducibility of apparent diffusion coefficient and intravoxel incoherent motion parameters at 3.0T. Journal of Magnetic Resonance Imaging : JMRI, 41(1): 149156.

16. Woo S, Lee JM, Yoon JH, Joo I, Han JK, Choi BI (2014) Intravoxel incoherent motion diffusion-weighted MR imaging of hepatocellular carcinoma: correlation with enhancement degree and histologic grade. Radiology, $\mathbf{2 7 0}$ (3): 758-767.

17. Edmondson HA and Steiner PE (1954) Primary carcinoma of the liver: a study of 100 cases among 48,900 necropsies. Cancer, 7(3): 462-503.

18. Tahir B, Sandrasegaran K, Ramaswamy R, Bertrand K, Mhapsekar R, Akisik FM, Saxena R (2011) Does the hepatocellular phase of gadobenate dimeglumine help to differentiate hepatocellular carcinoma in cirrhotic patients according to histological grade? Clinical Radiology, 66(9): 845-852.

19. Channual S, Tan N, Siripongsakun S, Lassman C, Lu DS, Raman SS (2015) Gadoxetate disodium-enhanced MRI to differentiate dysplastic nodules and grade of hepatocellular carcinoma: Correlation with histopathology. AJR American Journal of Roentgenology, 205(3): 546-553.

20. Coleman WB (2003) Mechanisms of human hepatocarcinogenesis. Current Molecular Medicine, 3(6): 573-588.

21. Guo W, Zhao S, Yang Y, Shao G (2015) Histological grade of hepatocellular carcinoma predicted by quantitative diffusion-weighted imaging. Int J Clin Exp Med, 8(3): 41644169.
22. Umeda S, Kanda M, Kodera Y (2018) Emerging evidence of molecular biomarkers in hepatocellular carcinoma. Histology and Histopathology, 33(4): 343-355.

23. Setshedi M, Andersson M, Kgatle MM, Roberts L (2018) Molecular and cellular oncogenic mechanisms in hepatocellular carcinoma. South African Medical Journal = Suid-Afrikaanse tydskrif vir geneeskunde, 108(8b): 41-46.

24. Ogihara $Y$, Kitazume $Y$, Iwasa $Y$, Taura $S$, Himeno $Y$, Kimura T, Sawano S, Terada S, Tanabe M, Saida Y, et al. (2018) Prediction of histological grade of hepatocellular carcinoma using quantitative diffusion-weighted MRI: a retrospective multivendor study. The British Journal of Radiology, 91(1084): 20170728.

25. Erra P, Puglia $M$, Ragozzino A, Maurea $S$, Liuzzi R, Sabino G, Barbuto L, Cuocolo A, Imbriaco M (2015) Appearance of hepatocellular carcinoma on gadoxetic acid-enhanced hepato-biliary phase MR imaging: a systematic review. $L a$ Radiologia Medica, 120(11): 1002-1011.

26. Sano K, Ichikawa T, Motosugi U, Sou H, Muhi AM, Matsuda M, Nakano M, Sakamoto M, Nakazawa T, Asakawa M et al. (2011) Imaging study of early hepatocellular carcinoma: usefulness of gadoxetic acid-enhanced MR imaging. Radiology, 261(3): 834-844.

27. Chou CT, Chou JM, Chang TA, Huang SF, Chen CB, Chen YL, Chen RC (2013) Differentiation between dysplastic nodule and early-stage hepatocellular carcinoma: the utility of conventional MR imaging. World Journal of Gastroenterology, 19(42): 7433-7439.

28. Asayama $\mathrm{Y}$, Yoshimitsu K, Nishihara $\mathrm{Y}$, Irie H, Aishima S, Taketomi A, Honda H (2008) Arterial blood supply of hepatocellular carcinoma and histologic grading: radiologic-pathologic correlation. AJR American Journal of Roentgenology, 190(1): W28-34.

29. Yasuda S, Arii S, Mori A, Isobe N, Yang W, Oe H, Fujimoto A, Yonenaga Y, Sakashita H, Imamura M (2004) Hexokinase II and VEGF expression in liver tumors: correlation with hypoxia-inducible factor 1 alpha and its significance. Journal of Hepatology, 40(1): 117-123. 
